\title{
Tarifvergütungen für berufsfachlich qualifizierte Beschäftigte
}

\author{
Die tariflichen Vergütungen weisen traditionell ein hohes Maß an Differenzierung zwi- \\ schen den Branchen auf. Der Beitrag analysiert Ausmaß und Entwicklungsdynamik dieser \\ Unterschiede anhand ausgewählter Industrie- und Dienstleistungssektoren und für Be- \\ schäftigtengruppen mit vergleichbarer beruflicher Qualifikation. Dabei wird deutlich, dass \\ sich der Einkommensvorsprung der Industrie seit Mitte der1990er Jahre vergrößert hat.
}

REINHARD BISPINCK, WSI-TARIFARCHIV

\section{Zielsetzung und Vorgehensweise}

Die Festlegung von Höhe und Differenzierung der (tariflichen) Arbeitseinkommen ist das Resultat einer ganzen Reihe unterschiedlicher Bestimmungsfaktoren. Dazu zählt die wirtschaftliche Leistungskraft einer Branche; d. h. die jeweiligen Wertschöpfungs-, Produktivitäts- und Gewinnentwicklungen bestimmen maßgeblich die Spielräume bei der Einkommensgestaltung. Die individuellen Arbeitseinkommen differenzieren sich des Weiteren nach Maßgabe der schulischen und beruflichen Ausgangsqualifikation bzw. der konkret im Arbeitsprozess abgeforderten Qualifikation. Daneben spielen Knappheitsverhältnisse am Arbeitsmarkt eine wichtige Rolle und schließlich sind auch institutionelle Faktoren wie die Ausgestaltung des Tarifsystems sowie der Organisationsgrad und das Durchsetzungsvermögen der Gewerkschaften von Bedeutung. Unabhängig von den genannten Dimensionen übt nicht zuletzt die gesellschaftliche Wertschätzung und Bewertung von Tätigkeitsfeldern und einzelnen Berufen einen erheblichen Einfluss auf Einkommenshöhe und -spreizung aus. Ein integraler Bestandteil dieser gesellschaftlichen Bewertung war auch die lange Zeit vorherrschende Vorstellung, dass ein Lohn so bemessen sein müsse, dass man (genauer: der Mann) davon auch seine Familie ernähren kann. Dieser Anspruch galt namentlich für den klassischen Tätigkeitsbereich des qualifizierten Industriearbeiters bzw. den Fachangestellten als Prototyp des männlichen Familienernährers. Für die Bereiche klassischer Frauentätigkeiten in der Industrie wie auch im Dienstleistungssektor ist anzunehmen, dass sie gerade deswegen ein niedrigeres Lohn- und Gehaltsniveau aufweisen.

Vor diesem Hintergrund werden im Folgenden die tariflichen Jahreseinkommen von Beschäftigten mit abgeschlossener Berufsausbildung und mit Fachhochschulqualifikation analysiert. ${ }^{\bullet}$ Dargestellt und verglichen werden das jeweilige tarifliche Einkommensniveau und seine Entwicklung im Zeitablauf. Dafür wurden Branchen und Tätigkeitsbereiche zugrunde gelegt, die Kernsektoren der Industrie, den privaten Dienstleistungssektor und den Bereich von Gesundheits-, Pflege-, Sozial- und Erziehungsdienstleistungen abdecken:

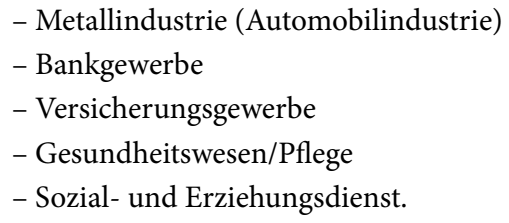

Die Auswertung erfolgt in Anlehnung an das Forschungsdesign des Projektes „Was kommt nach dem Familienlohn? Probleme und Möglichkeiten der Regulierung von Einkommensrisiken bei Normalarbeitnehmerlnnen im Ländervergleich" (Projekt-Nr. 2010-375-4), das seit 2011 (bis 2013) von der Hans-Böckler-Stiftung gefördert wird. Wir danken Gabriele Gröschl-Bahr und Detlef Raabe von ver.di für wertvolle Unterstützung bei der Zusammenstellung der Daten. 
In der Metallindustrie wurde der Tarifbereich Nordwürttemberg/Nordbaden ausgewählt. Im Banken- und Versicherungsgewerbe bestehen jeweils bundesweite Tarifverträge. Im Bereich Gesundheit/Pflege, Sozial- und Erziehungsdienst wurden neben dem öffentlichen Dienst (Gemeinden) auch ausgewählte Wohlfahrtsverbände berücksichtigt.

Bei den Tarifeinkommen wurden die Grundvergütungen sowie als regelmäßig gezahlte Einkommensbestandteile das Urlaubsgeld und die Jahressonderzahlung (Weihnachtsgeld) berücksichtigt. Nicht einbezogen wurden in der Regel variable Zulagen und Zuschläge, die jeweils von der konkreten Arbeitsleistung, von besonderen Arbeitszeitformen (Überstunden, Nacht- und Schichtarbeit) oder spezifischen Funktionen abhängen. Eine Ausnahme bildet die tarifliche Leistungszulage in der Metallindustrie (vgl. Abschnitt 2.1). Pauschal- und Einmalzahlungen blieben ebenso unberücksichtigt wie Tarifveränderungen im Laufe der jeweiligen Jahre. Die Jahreseinkommen wurden auf Basis der zum jeweiligen Jahresende gültigen Tarifvergütungen berechnet.

Als Qualifikations- bzw. Anforderungsstufen wurden soweit möglich - zwei Ausprägungen des „Normalarbeitnehmers" berücksichtigt:

- Beschäftigte mit abgeschlossener, in der Regel dreijähriger Berufsausbildung, etwa im Rahmen des dualen Systems der beruflichen Bildung oder als vollzeitschulische Ausbildung. - Beschäftigte mit einem Fachhochschulabschluss oder einer vergleichbaren Qualifikation.

In den einschlägigen Lohn-, Gehalts- und Entgelttarifverträgen für die genannten Branchen wurden jeweils die Einstiegsgruppe sowie die oberste erreichbare Vergütungsgruppe für die beiden Beschäftigtengruppen identifiziert. Auf diese Weise können Einkommenskorridore abgebildet werden, in denen sich die Beschäftigten mit dieser Qualifikation bewegen. Die Analyse wurde auf die westdeutschen Tarifgebiete beschränkt, um eventuelle Auswirkungen des deutsch-deutschen Einigungsprozesses auf die Tarifentwicklung auszuschließen. ${ }^{2}$

Der Analysezeitraum erstreckt sich von 1995 bis 2010. Die Einkommensdaten werden in Fünfjahresabschnitten wiedergegeben. Für den Bereich der Wohlfahrtsverbände beschränken sich die Angaben auf den aktuellen Rand.

In allen untersuchten Gehaltstarifverträgen und auch in den neuen entgeltbezogenen Vergütungstarifverträgen gibt es Differenzierungen, die an die Dauer der Tätigkeit im Betrieb bzw. innerhalb des Berufs/der Tätigkeit oder auch in der konkreten Tarifgruppe anknüpfen. Eine Senioritätsorientierung, die direkt an das Lebensalter anknüpfte, bestand lediglich im früheren Bundesangestelltentarifvertrag (BAT).

In einzelnen Tarifbereichen waren in früheren Jahren unmittelbar familienbezogene Einkommenskomponenten Bestandteile des Tarifeinkommens:

- Im BAT gab es bis zu seiner Ablösung durch den Tarifvertrag für den öffentlichen Dienst (TVöD) im Jahr 2005 den sogenannten Ortszuschlag. Der Ortszuschlag war, anders als der Name nahelegt, ein familienbezogener Vergütungsbestandteil, differenziert nach Familienstand, Kinderzahl und Vergütungsgruppe.

- Im Bankgewerbe sah der Tarifvertrag bis 1995 eine Kinderzulage vor von zuletzt 25/50 DM (12,78/25,56€) für ein Kind bzw. zwei oder mehr Kinder. Sie wird seit 1996 als Besitzstandssicherung weitergezahlt.

Eine vergleichbare Regelung stellte im Versicherungsgewerbe der Sozialzuschlag dar. Er betrug zuletzt 18/35 DM für ein Kind bzw. zwei und mehr Kinder und wird seit 1995 ebenfalls nur noch als Besitzstand (10/20 €) gezahlt.

In der Metallindustrie existier(t)en vergleichbare familienbezogene Tarifbestandteile nicht.

Anders als die direkt familienbezogenen Entgeltbestandteile können berufserfahrungs- und altersabhängige Einkommenselemente allenfalls sehr indirekt als solche interpretiert werden, und zwar lediglich insofern, als mit steigendem Lebensalter (und wachsender Berufserfahrung) auch die Wahrscheinlichkeit größer wird, eine Familie zu gründen.

\section{Branchenbezogene Daten}

Im Folgenden werden die Befunde aus den ausgewählten Branchen und Tarifbereichen nach Qualifikationsniveaus bzw. Tätigkeiten differenziert dargestellt. Eine branchenvergleichende Analyse schließt sich an.

\subsection{Metallindustrie}

Die Metallindustrie ist die industrielle Kernbranche der bundesdeutschen Wirtschaft. Die Tarifverträge regeln die Einkommens- und Arbeitsbedingungen für die zahlreichen Teilbranchen der Metall- und Elektroindustrie, darunter auch die Automobilindustrie. Nur für die Volkswagen AG besteht nach wie vor ein Haustarifvertrag, der sich zunehmend am Niveau der Flächentarifverträge orientiert. Der Tarifbereich Nordwürttemberg-Nordbaden ist von herausgehobener Bedeutung, da hier in der Vergangenheit zahlreiche Pilotabschlüsse getätigt wurden. Auch die Automobilindustrie spielt eine zentrale Rolle: In diesem Tarifbereich sind beispielsweise zentrale Produktionsstandorte der Daimler AG angesiedelt.

Für die Tarifanalyse sind folgende Gesichtspunkte zu beachten: Im Jahr 2002 wurde die Einführung eines neuen Entgeltrahmenabkommens (ERA) beschlossen, das die bis dahin geltenden getrennten Lohn- und Gehaltstarifverträ-

(2) Grundsätzlich gelten die gefundenen Ergebnisse auch für die ostdeutschen Tarifbereiche, denn die tariflichen Vergütungsstrukturen ähneln den westdeutschen weitgehend. 
ge durch einen einheitlichen Entgelttarifvertrag ablöste. Als Einführungszeitraum wurde der Zeitraum vom 1.3.2005 bis zum 29.2.2008 festgelegt. Im Zusammenhang mit der ERAEinführung wurden auch sogenannte ERA-Strukturkomponenten vereinbart, die bei der Berechnung des tariflichen Jahresverdienstes 2005 berücksichtigt wurden. Die von der IG Metall bis 2010 fortgeführten Lohn- und Gehaltstabellen dürften zwar laut Tarifvertrag in der Praxis keine Rolle mehr spielen, werden aber für das Jahr 2010 nachrichtlich noch einmal ausgewiesen.

Die Berechnungen (Tabelle 1) berücksichtigen die tariflichen Grundvergütungen, die tariflich garantierten Leistungszulagen sowie das tarifliche Urlaubsgeld und die Jahressonderzahlung. In den alten Vergütungstarifverträgen bestanden zwölf Lohngruppen und jeweils sieben Gehaltsgruppen für die kaufmännischen und technischen Angestellten. Das einheitliche Entgeltrahmenabkommen für alle Beschäftigten umfasst 17 Entgeltgruppen. Für die Gruppe der Beschäftigten mit abgeschlossener Berufsausbildung wurden folgende Vergütungsgruppen zugrunde gelegt:

- Arbeiter: Lohngruppen 7 und 12,

- kaufmännische Angestellte: Gehaltsgruppen K 2 (Eingangsstufe) und K 4 (Endstufe),

- technische Angestellte: Gehaltsgruppe T 2 (Eingangsstufe) und $\mathrm{T} 4$ (Endstufe),
- Arbeitnehmer: Entgeltgruppen 7 und 9 (für das Jahr 2010, nach ERA-Einführung).

Für die Beschäftigten mit Fachhochschulqualifikation wurden folgende Gruppen herangezogen:

- kaufmännische Angestellte: Gehaltsgruppen K 4 (Eingangsstufe) und $\mathrm{K} 7$ (Endstufe),

- technische Angestellte: Gehaltsgruppe T 4 (Eingangsstufe) und $\mathrm{T} 7$ (Endstufe),

- Arbeitnehmer: Entgeltgruppen 12 und 17 (für das Jahr 2010, nach ERA-Einführung).

Die garantierte tarifliche Leistungszulage beträgt nach dem Entgeltrahmenabkommen im Betriebsdurchschnitt $15 \%$, die Leistungszulagen für Arbeiter bzw. Angestellte beliefen sich auf 16 bzw. $10 \%$ im Durchschnitt. Ein individueller Anspruch bestand und besteht nicht.

Die Einkommensentwicklung verlief in den verschiedenen tariflichen Vergütungsgruppen im Berichtszeitraum gleichmäßig. Legt man die jahresbezogenen Tarifvergütungen auf Basis der Lohn- und Gehaltstabellen bis einschließlich 2010 zugrunde, errechnet sich für 1995-2010 ein einheitlicher Anstieg um 45,0 \%. Das Bild verändert sich durch Einbeziehung der neuen Entgelttabellen für das Jahr 2010. Im Facharbeiterbereich liegt das Eingangsentgelt in

TABELLE 1

Tarifliche Jahresgrundvergütung inkl. Urlaubsgeld und Jahressonderzahlung - Metallindustrie Nordwürttemberg/Nordbaden -

Angaben in Euro

\begin{tabular}{|c|c|c|c|c|c|c|c|}
\hline Tätigkeit & Qualifikation/Anforderung & Vergütungsgruppe & 1995 & 2000 & 2005 & 2010 & 2010 pro Monat \\
\hline Facharbeiter & $\begin{array}{l}\text { Berufsausb. } \\
\text { Berufsausb. }\end{array}$ & $\begin{array}{l}\text { LG } 7 \\
\text { LG } 12\end{array}$ & $\begin{array}{l}23.006 \\
31.063\end{array}$ & $\begin{array}{l}25.544 \\
34.488\end{array}$ & $\begin{array}{l}28.755 \\
38.825\end{array}$ & $\begin{array}{l}33.356 \\
45.037\end{array}$ & $\begin{array}{l}2.780 \\
3.753\end{array}$ \\
\hline kaufm. Angestellte & $\begin{array}{l}\text { Berufsausb. } \\
\text { Berufsausb. } \\
\text { FH-Abschluss } \\
\text { FH-Abschluss }\end{array}$ & $\begin{array}{l}\text { K } 2 \text { (1. Bj.) } \\
\text { K } 4 \text { (4. Bj.) } \\
\text { K } 4 \text { (1. Bj.) } \\
\text { K } 7\end{array}$ & $\begin{array}{l}21.292 \\
34.078 \\
29.502 \\
46.590\end{array}$ & $\begin{array}{l}23.642 \\
37.842 \\
32.756 \\
51.722\end{array}$ & $\begin{array}{l}26.619 \\
42.606 \\
36.875 \\
58.231\end{array}$ & $\begin{array}{l}30.878 \\
49.422 \\
42.774 \\
67.547\end{array}$ & $\begin{array}{l}2.573 \\
4.119 \\
3.564 \\
5.629\end{array}$ \\
\hline techn. Angestellte & $\begin{array}{l}\text { Berufsausb. } \\
\text { Berufsausb. } \\
\text { FH-Abschluss } \\
\text { FH-Abschluss }\end{array}$ & $\begin{array}{l}\text { T } 2 \text { (1. Bj.) } \\
\text { T } 4 \text { (4. Bj.) } \\
\text { T } 4 \text { (1. Bj.) } \\
\text { T } 7\end{array}$ & $\begin{array}{l}24.059 \\
37.431 \\
32.944 \\
51.017\end{array}$ & $\begin{array}{l}26.710 \\
41.565 \\
36.584 \\
56.651\end{array}$ & $\begin{array}{l}30.068 \\
46.793 \\
41.185 \\
63.782\end{array}$ & $\begin{array}{l}34.879 \\
54.280 \\
47.774 \\
73.986\end{array}$ & $\begin{array}{l}2.907 \\
4.523 \\
3.981 \\
6.165 \\
\end{array}$ \\
\hline Arbeitnehmer/in & $\begin{array}{l}\text { Berufsausb. } \\
\text { Berufsausb. } \\
\text { Berufsausb. } \\
\text { Experten-/Spezialistentätigkeit } \\
\text { Berufsausb. } \\
\text { Experten-/Spezialistentätigkeit } \\
\text { FH-Abschluss } \\
\text { FH-Abschluss }\end{array}$ & $\begin{array}{l}\text { E } 7 \\
\text { E } 9 \\
\text { E } 10 \\
\text { E } 12 \\
\text { E } 12 \\
\text { E } 17\end{array}$ & & & & $\begin{array}{l}38.544 \\
43.941 \\
46.834 \\
53.381 \\
53.381 \\
71.888\end{array}$ & $\begin{array}{l}3.212 \\
3.662 \\
3.903 \\
4.448 \\
4.448 \\
5.991\end{array}$ \\
\hline
\end{tabular}


der EG 7 mit fast $38.600 €$ über der LG 7 des alten Lohntarifvertrages mit ca. $33.400 €$. In der Endstufe erreichten die Facharbeiter in der LG 12 rund $45.000 €$, als Spezialisten gelangen sie in der EG 12 bis zu $53.400 €$.

\subsection{Bank- und Versicherungsgewerbe}

Für die beiden Branchen des Finanzdienstleistungssektors bestehen mehrere getrennte Tarifvertragswerke. Ausgewertet wurden die Tarifverträge für das private und öffentliche Bankgewerbe (ohne die im TVöD gesondert geregelten Sparkassen) und für das private Versicherungsgewerbe ( $\mathrm{Ta}$ belle 2). In beiden Branchen bestehen bundesweit geltende Tarifverträge.

Der Tarifvertrag für das Bankgewerbe sieht neun Gehaltsgruppen vor. Die Gruppe 4 ist die mittlere Gruppe und dient als Einstiegsgruppe für Beschäftigte mit abgeschlossener Berufsausbildung, also auch Bankkaufleute. Sie können in der beruflichen Praxis mit entsprechender Fortbildung bis zur obersten Gehaltsgruppe 9 vordringen. Eine untere Einstiegsgruppe für Beschäftigte mit Fachhochschulqualifikation lässt sich nach Angaben der gewerkschaftlichen Praktiker nicht exakt benennen.

Im Tarifvertrag für das Versicherungsgewerbe sieht die Gehaltstabelle acht Vergütungsgruppen vor. Die Gehaltsgruppe III ist die Einstiegsgruppe für Beschäftigte mit abgeschlossener Berufsausbildung, also auch für Versicherungskaufleute, die - wie die Bankkaufleute - die oberste Gehaltsgruppe, in diesem Fall die Gruppe VIII, erreichen können.

Neben der tariflichen Grundvergütung wurden im Bankgewerbe die Jahressonderzahlung mit aktuell $100 \%$ eines Monatsentgeltes sowie im Versicherungsgewerbe das Urlaubsgeld und die Jahressonderzahlung mit 50 bzw. 80 \% eines Monatsentgeltes berücksichtigt.

Damit ergibt sich für die beiden Branchen folgendes Bild: Bankkaufleute starten im Jahr 2010 mit einem Jahresgehalt von rund $28.000 €(2.335 € /$ Monat $)$ und können in der obersten Tarifgruppe rund $54.500 €(4.544 € /$ Monat) erreichen. Dies markiert zugleich die tarifliche Obergrenze für Beschäftigte mit Fachhochschulqualifikation. Versiche- rungskaufleute erhalten $2010 \mathrm{zu}$ Beginn mit $29.800 €$ rund $1.800 €$ mehr und auch ihre oberste erreichbare Tarifvergütung liegt mit knapp $56.000 €(2.485 €$ bzw. $4.665 € /$ Monat $)$ über dem Niveau des Bankgewerbes.

Die Tarifentwicklung verlief in beiden Branchen parallel: Im Bankgewerbe stiegen die hier ausgewiesenen tariflichen Jahreseinkommen von 1995 bis 2010 um 33,8 \%, im Versicherungsgewerbe waren es im gleichen Zeitraum $33,3 \%$.

\section{3 Öffentlicher Dienst}

Der öffentliche Dienst, namentlich die Gemeinden, sind der größte Arbeitgeber für Beschäftigte in Gesundheits-, Sozial- und Erziehungsberufen. Grundlage unserer Analyse für diese Berufsgruppen sind daher die Tarifverträge für diesen Tarifbereich. Die Tarifstrukturen im Bereich der Gemeinden wurden bis 2005 durch den BAT geprägt. Im Jahr 2005 vereinbarten die Gewerkschaften des öffentlichen Dienstes unter Federführung der Vereinten Dienstleistungsgewerkschaft (ver.di) mit den Arbeitgebern den TVöD, der die bis dahin geltenden Lohntarifverträge und den BAT in einem neuen einheitlichen Entgelttarifvertrag für alle Beschäftigten zusammenfasste. Die folgenden Daten (Tabelle 3) zu den tariflichen Vergütungen beziehen sich für die Jahre 1995 und 2000 auf den BAT und für die Jahre 2005 und 2010 auf den TVöD. Im Sozial- und Erziehungsdienst wurde 2009 im Rahmen des TVöD eine eigenständige Entgelttabelle vereinbart, die für 2010 berücksichtigt wurde.

Um beide Qualifikationsniveaus, berufliche Ausbildung und Fachhochschulabschluss, mit Einstiegsniveau und oberer Vergütungsgrenze zu berücksichtigen, wurden die folgenden vier Berufe mit den entsprechenden Tarifgruppen in die Auswertung einbezogen:

- Altenpfleger/Altenpflegerin: BAT Kr. IV und VI sowie TVöD EG 7a Stufe 1 und EG 9b Endstufe,

- Krankenschwester/pfleger: BAT Kr. IV und Kr. X sowie TVöD Kr. 7a Stufe 1 und Kr. 11a Endstufe,

TABELLE 2

Tarifliche Jahresgrundvergütung inkl. Urlaubsgeld und Jahressonderzahlung - Banken- und Versicherungsgewerbe Angaben in Euro

\begin{tabular}{|c|c|c|c|c|c|c|c|}
\hline Tätigkeit & Qualifikation/Anforderung & Vergütungsgruppe & 1995 & 2000 & 2005 & 2010 & 2010 pro Monat \\
\hline \multirow[t]{2}{*}{ Bankkaufmann/frau } & Berufsausb. & 4 (Anf.stufe) & 20.924 & 23.091 & 26.143 & 28.015 & 2.335 \\
\hline & Berufsausb. & 9 (Endstufe) & 40.745 & 44.972 & 50.895 & 54.522 & 4.544 \\
\hline \multirow{2}{*}{$\begin{array}{l}\text { Versicherungskauf- } \\
\text { mann/frau }\end{array}$} & Berufsausb. & 3 (Anf.stufe) & 22.366 & 24.583 & 26.972 & 29.819 & 2.485 \\
\hline & Berufsausb. & 8 (Endstufe) & 41.984 & 46.160 & 50.646 & 55.980 & 4.665 \\
\hline
\end{tabular}


TABELLE 3

Tarifliche Jahresgrundvergütung inkl. Urlaubsgeld und Jahressonderzahlung - Öffentlicher Dienst Gemeinden West -

Angaben in Euro

\begin{tabular}{|c|c|c|c|c|c|c|c|}
\hline Tätigkeit & Qualifikation/Anforderung & Vergütungsgruppe & 1995 & 2000 & 2005 & 2010 & 2010 pro Monat \\
\hline \multirow[t]{6}{*}{ Altenpfleger/in } & \multirow[t]{3}{*}{$\begin{array}{l}\text { mit staatl. Anerkennung/ } \\
\text { Abschlussprüfung }\end{array}$} & $\begin{array}{l}\text { BAT Kr. IV } \\
\text { ledig }\end{array}$ & 21.912 & 23.507 & & & \\
\hline & & verh., $2 \mathrm{Ki}$. & 25.132 & 26.965 & & & \\
\hline & & TVöD EG 7a, St. 1 & & & 23.865 & 26.289 & 2.191 \\
\hline & \multirow[t]{3}{*}{$\begin{array}{l}\text { mind. } 10 \text { Pflegepersonen } \\
\text { unterstellt }\end{array}$} & $\begin{array}{l}\text { BAT Kr. VI } \\
\text { ledig }\end{array}$ & 29.376 & 31.534 & & & \\
\hline & & verh. $2 \mathrm{Ki}$. & 32.596 & 34.992 & & & \\
\hline & & $\begin{array}{l}\text { TVöD EG 9b, } \\
\text { Endstufe }\end{array}$ & & & 36.352 & 39.677 & 3.306 \\
\hline \multirow[t]{3}{*}{ Erzieher/in } & \multirow[t]{3}{*}{ mit staatl. Anerkennung } & $\begin{array}{l}\text { BAT VI b/V c } \\
\text { ledig }\end{array}$ & 21.231 & 22.769 & & & \\
\hline & & verh., $2 \mathrm{Ki}$. & 24.451 & 26.227 & & & \\
\hline & & TVöD EG 6/S 6 St. 2 & & & 25.284 & 29.243 & 2.437 \\
\hline \multirow[t]{4}{*}{ Leiter/in Kita } & \multirow{4}{*}{$\begin{array}{l}\text { ab } 40 \text { Plätze } \\
\text { mind. } 180 \text { Ki. }\end{array}$} & TVöD S10, Stufe 6 & & & & 44.819 & 3.735 \\
\hline & & $\begin{array}{l}\text { BAT III } \\
\text { ledig }\end{array}$ & 42.433 & 45.577 & & & \\
\hline & & verh., 2 Ki. & 45.658 & 49.045 & & & \\
\hline & & TVöD E 11/S 17 & & & 49.088 & 53.563 & 4.464 \\
\hline \multirow[t]{3}{*}{ Krankenschwester/-pfleger } & \multirow[t]{3}{*}{ mit staatl. Anerkennung } & $\begin{array}{l}\text { BAT Kr. IV } \\
\text { ledig }\end{array}$ & 21.912 & 23.507 & & & \\
\hline & & verh., $2 \mathrm{Ki}$. & 25.132 & 26.965 & & & \\
\hline & & $\begin{array}{l}\text { TVöD Kr. 7a } \\
\text { St. } 1\end{array}$ & & & 23.865 & 26.612 & 2.218 \\
\hline \multirow[t]{3}{*}{$\begin{array}{l}\text { Krankenschwester/-pfleger } \\
\text { (Pflegedienstleitung) }\end{array}$} & \multirow[t]{3}{*}{$\begin{array}{l}\text { mind. } 200 \text { Pflege- } \\
\text { personen unterstellt }\end{array}$} & $\begin{array}{l}\text { BAT Kr. X } \\
\text { ledig }\end{array}$ & 37.830 & 40.628 & & & \\
\hline & & verh., 2 Ki. & 41.106 & 44.147 & & & \\
\hline & & $\begin{array}{l}\text { TVöD Kr.11a } \\
\text { Endstufe }\end{array}$ & & & 46.528 & 50.912 & 4.243 \\
\hline \multirow[t]{6}{*}{ Sozialarbeiter/in } & \multirow[t]{3}{*}{ mit staatl. Anerkennung } & $\begin{array}{l}\text { BAT V b/lV b (V b) } \\
\text { ledig }\end{array}$ & 24.080 & 25.842 & & & \\
\hline & & verh., $2 \mathrm{Ki}$. & 27.356 & 29.361 & & & \\
\hline & & $\begin{array}{l}\text { TVöD EG } 9 \\
\text { St. } 2 \text { / S } 11 \text { St. } 2\end{array}$ & & & 29.312 & 33.679 & 2.807 \\
\hline & \multirow[t]{3}{*}{ oberste Gruppe } & $\begin{array}{l}\text { BAT II } \\
\text { ledig }\end{array}$ & 47.535 & 51.064 & & & \\
\hline & & verh., $2 \mathrm{Ki}$. & 50.812 & 54.583 & & & \\
\hline & & TVöD E 12 / S 18 & & & 53.760 & 58.615 & 4.885 \\
\hline
\end{tabular}

Quelle:WSI-Tarifarchiv.

- Erzieher/Erzieherin: BAT VI b/V c sowie TVöD EG 6 / S 6 Stufe 2,

- Leiter/Leiterin einer Kindertagesstätte: BAT III bzw. TVöD

E 11 / S 17,

- Sozialarbeiter/Sozialarbeiterin: BAT V b/IV b und BAT II sowie TVöD EG 9 Stufe 2 / S 11 Stufe 2 und E 12 / S 18
Die wesentlichen Ergebnisse bezogen auf das Jahr 2010 lassen sich folgendermaßen zusammenfassen (Tabelle 3):

- Altenpfleger/Altenpflegerinnen mit staatlicher Anerkennung starten 2010 mit rund $26.300 €$, unterstehen ihnen mindestens 10 Pflegekräfte steigt ihre Tarifvergütung in der Endstufe auf knapp $39.700 €$ 
- Krankenschwestern/-pfleger mit abgeschlossener Ausbildung beginnen 2010 mit $26.600 €$. In der Position der Pflegeleitung mit mindestens 200 unterstellten Pflegepersonen erreicht ihre Jahresvergütung in der Endstufe etwa $50.900 €$. Voraussetzung für eine solche Leitungsposition ist allerdings eine ein- bis zweijährige Weiterbildung.

- Erzieher/Erzieherinnen verdienten 2010 zu Beginn ihrer Tätigkeit rund $29.250 €$, ein Leiter/eine Leiterin einer Kindertagesstätte mit mindestens 180 Kindern erreicht ein maximales Tarifeinkommen von knapp $53.600 €$.

- Sozialarbeiter/Sozialarbeiterinnen erhalten in der untersten Tarifgruppe im Jahr knapp $33.700 €$ in der Endstufe der obersten erreichbaren Tarifgruppe bekommen sie eine Vergütung von gut $58.600 €$.

In Bezug auf die Einkommensentwicklung seit 1995 ist vor allem die Wirkung des Ortszuschlages, eine im alten BAT in Abhängigkeit vom Familienstand und der Kinderzahl abhängige Einkommenskomponente (siehe oben), zu berücksichtigen: Für die Jahre 1995 und 2000 wurden daher die Tarifvergütungen sowohl für ledige Beschäftigte als auch für Beschäftigte mit zwei Kindern berechnet. Je niedriger die Grundvergütung, umso höher die relative Wirkung des höheren Ortszuschlages. Bezogen auf das Jahr 1995 verdienen Beschäftigte mit zwei Kindern je nach Tarifgruppe zwischen 3.220 und $3.276 €$ mehr als vergleichbare ledige Beschäftigte ohne Kinder. Beschäftigte mit zwei Kindern erhalten damit zwischen 6,4 und 14,1\% mehr als ledige Beschäftigte ohne Kinder.

TABELLE 4

\section{Tarifliche Jahresvergütung Wohlfahrtsverbände für Altenpfleger/ Altenpflegerinnen}

Angaben in Euro

\begin{tabular}{|c|c|c|c|c|c|}
\hline TV / AVR & Anwender & Tarifgebiet & $\begin{array}{l}\text { Vergütungs- } \\
\text { gruppe }\end{array}$ & Jahr & Monat \\
\hline \multicolumn{6}{|l|}{$\begin{array}{l}\text { Eingangsstufe } \\
\text { (Berufsanfänger/in) }\end{array}$} \\
\hline TV AWO NRW & AWO NRW & West & $\mathrm{Kr} 7 \mathrm{a}$ & 26.477 & 2.206 \\
\hline AVB & Paritätischer & West & Gr D & 27.420 & 2.285 \\
\hline AVR Caritas & Caritas/Malteser & West & $\mathrm{Kr} 7 \mathrm{a}$ & 27.609 & 2.301 \\
\hline DRK-Reformtarif & DRK & bundesweit & $\mathrm{Kr} 7 \mathrm{a}$ & 27.905 & 2.325 \\
\hline AVR DW Bayern & Diakonie Bayern & West & EG 8 & 31.023 & 2.585 \\
\hline \multicolumn{6}{|l|}{$\begin{array}{l}\text { maximale } \\
\text { Erfahrungsstufe }\end{array}$} \\
\hline AVB & Paritätischer & West & Gr D & 32.340 & 2.695 \\
\hline AVR DW Bayern & Diakonie Bayern & West & EG 8 & 34.384 & 2.865 \\
\hline TV AWO NRW & AWO NRW & West & $\mathrm{Kr} 7 \mathrm{a}$ & 36.252 & 3.021 \\
\hline AVR Caritas & Caritas/Malteser & West & $\mathrm{Kr} 7 \mathrm{a}$ & 37.498 & 3.125 \\
\hline DRK-Reformtarif & DRK & bundesweit & $\mathrm{Kr} 7 \mathrm{a}$ & 37.786 & 3.149 \\
\hline
\end{tabular}

Quelle: Wohlfahrtintern-Tarifarchiv.
Die Tarifentwicklung von 1995 bis 2010 belief sich im Durchschnitt auf eine Steigerung von knapp $23 \%$. Allerdings bestanden erhebliche Unterschiede je nach Beruf, Tarifgruppe und Familienstand bzw. Kinderzahl. Die folgenden Beispiele zeigen eine Spannweite zwischen 4,6 \% und 39,9\%:

- Für ledige Altenpfleger/Altenpflegerinnen in der Eingangsgruppe stiegen die Tarifvergütungen in diesem Zeitraum um rund $4.380 €$, das entspricht einem Anstieg um 20,0\%, für dieselben Beschäftigten mit zwei Kindern beträgt die Steigerung aufgrund des höheren Ausgangsniveaus $1.160 €$ bzw. 4,6\%.

- Bei den Erziehern/Erzieherinnen beträgt die Steigerung für ledige rund $8.000 €$, für Beschäftigte mit zwei Kindern dagegen $4.790 €$. Das entspricht einem Anstieg von 37,7 bzw. $19,6 \%$.

- Die Sozialarbeiter/Sozialarbeiterinnen in der untersten Gruppe verzeichneten als Ledige einen Zuwachs von $9.600 €$, mit zwei Kindern dagegen rd. $6.320 €$, das heißt, der prozentuale Anstieg betrug 39,9 bzw. 23,1\%.

Bei dieser Berechnung des Tarifanstiegs ist grundsätzlich zu berücksichtigen, dass für Beschäftigte, die nach den Bestimmungen des BAT eingestellt wurden, Besitzstandsregelungen gelten. Hier dagegen handelt es sich um Einkommensvergleiche unter Annahme der Neueinstellung, also ohne Berücksichtigung der Besitzstandsregelungen.

\subsection{Wohlfahrtsverbände}

Die Wohlfahrtsverbände und ihre Einrichtungen spielen als Arbeitgeber im Bereich der sozialen Dienstleistungen eine große Rolle. Die Arbeits- und Einkommensbedingungen werden dort in Tarifverträgen (AWO, Deutsches Rotes Kreuz, Paritätischer Wohlfahrtsverband) und bei den kirchlichen Trägern (Caritas, Diakonie) durch Arbeitsvertragsrichtlinien (AVR) im Rahmen des sogenannten Dritten

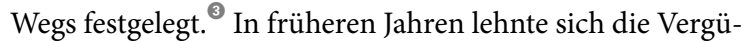
tung der Beschäftigten stark an den Tarifbedingungen für den öffentlichen Dienst an. Die Tarif- und Vergütungslandschaft hat sich in diesem Bereich jedoch in den vergangenen Jahren stark aufgefächert. Eine Darstellung über den gesamten Zeitraum 1995-2010 ist aufgrund der vorhandenen Datenlage nicht möglich. Im Folgenden werden anhand von wenigen ausgewählten (Tarif-)Bereichen die aktuellen Vergütungen im Bereich der Altenpflege dargestellt. Die Be-

3 Im kirchlichen Bereich besteht ein eigenständiges kollektives Arbeitsrecht, das auf dem verfassungsrechtlich abgesicherten Selbstbestimmungs- und Selbstverwaltungsrecht der Kirchen beruht. Die einvernehmliche Gestaltung der Arbeitsvertragsrichtlinien und der Vergütung in paritätisch besetzten Kommissionen wird als "Dritter Weg" bezeichnet. Er wird anstelle des herkömmlichen Tarifvertragssystems ( „Zweiter Weg“) bzw. der selbstständigen Setzung durch den Arbeitgeber ( „Erster Weg“) angewendet. 
rechnungen erfolgten auf Basis des Tarifstandes vom September 2012 und enthalten auch Einmalzahlungen und feste Zulagen.

- Die gelernten Altenpfleger/Altenpflegerinnen erzielen in der Eingangsstufe eine (tarifliche) Jahresvergütung zwischen rund 26.500 und $31.000 €$, das entspricht einem Monatseinkommen von rund 2.200 und knapp $2.600 €$. In der maximalen Erfahrungsstufe bewegt sich die Jahresvergütung zwischen gut 32.300 und $37.800 €$ oder 2.700 und $3.150 €$ pro Monat (Tabelle 4).

- Für den Bereich der Pflegedienstleitungen ergibt sich folgendes Bild (Tabelle 5): In der Eingangsstufe reichen die Jahresvergütungen von rund 35.400 bis zu $38.300 €$ (2.950 - $3.200 € \mathrm{im}$ Monat), in der höchsten Erfahrungsstufe besteht eine Spannweite von knapp 36.600 bis $44.200 €$ (3.050 - 3.680 € im Monat).

\section{Branchenergebnisse im Vergleich}

Die wesentlichen Ergebnisse der branchenspezifischen Tarifanalysen für die beiden Qualifikationsgruppen lassen sich folgendermaßen zusammenfassen. Bei Tätigkeiten mit abgeschlossener Berufsausbildung bewegt sich das tarifliche Jahreseinkommen zwischen rund $26.290 €$ im Einstiegsbereich und knapp $56.000 €$ im obersten Vergütungsbereich. Bei Tätigkeiten mit Fachhochschulqualifikation liegt der Tarifverdienst zwischen $33.700 €$ im Einstiegsbereich und knapp $71.900 €$ bei den obersten Vergütungen (Abbildung 1).

- Beschäftigte mit abgeschlossener Berufsausbildung: Die Spannweite der tariflichen Jahresvergütungen von Beschäftigten mit abgeschlossener Berufsausbildung reicht in den untersuchten Branchen im Jahr 2010 von rund 26.290 bis $56.000 €$. Das entspricht einer monatlichen Tarifvergütung zwischen rund 2.190 und $4.670 €$ (Tabelle 6).

- Bei der Einstiegsvergütung reicht die Bandbreite des Jahreseinkommens von rund $26.300 €$ bis zu $38.550 €$. Die höchste in den untersuchten Branchen gezahlte Einstiegsvergütung liegt damit $47 \%$ über der niedrigsten. Die Gesundheitsberufe liegen am unteren Ende der Skala, gefolgt von den Bankkaufleuten, den Erziehern und Erzieherinnen und den Versicherungskaufleuten. An der Spitze stehen die Metallbeschäftigten.

- Bei der obersten erreichbaren Vergütung für Beschäftigte mit abgeschlossener Ausbildung reicht die Spanne von $39.700 €$ bis zu knapp $56.000 €$, sie beträgt damit rund $41 \%$. Am unteren Ende stehen wiederum die Gesundheitsberufe, am oberen Ende schieben sich die Bank- und Versicherungskaufleute vor die Beschäftigten der Metallindustrie. Auch die höchste Eingruppierung im Erziehungsbereich liegt etwas über der Metallvergütung. Grundsätzlich ist bei den Vergütungen im Gesundheits- und Pflegebereich zu berück-
ABB. 1

\section{Spannweite der tariflichen Jahresvergütung nach Ausbildungsabschluss in ausgewählten Bereichen 2010}

Angaben in Euro Einstiegsvergütung $\mathbf{\square}$ oberste Vergütung
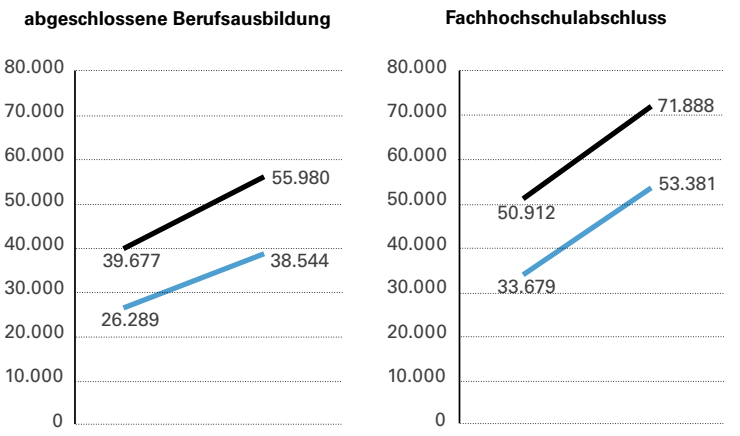

Quelle: WSI-Tarifarchiv (2010).

Mitteilungen

sichtigen, dass die obersten Vergütungen nur mit einer einbis zweijährigen Weiterbildung erreichbar sind. Tatsächlich werden viele dieser Positionen in der Praxis auch von Beschäftigten mit einer FH-Qualifikation besetzt.

- Beschäftigte mit Fachhochschulabschluss: Bei Beschäftigten mit Fachhochschulabschluss reicht das Spektrum von rund 33.700 bis $71.900 €$, das entspricht einem Monatsein-

\section{TABELLE 5}

Tarifliche Jahresvergütung Wohlfahrtsverbände Pflegedienstleitung Angaben in Euro

\begin{tabular}{|c|c|c|c|c|c|}
\hline TV / AVR & Anwender & Tarifgebiet & $\begin{array}{l}\text { Vergütungs- } \\
\text { gruppe }\end{array}$ & Jahr & Monat \\
\hline \multicolumn{6}{|l|}{$\begin{array}{l}\text { Eingangsstufe } \\
\text { (Berufsanfänger/in) }\end{array}$} \\
\hline AVB & Paritätischer & West & GrE & 32.340 & 2.695 \\
\hline TV AWO NRW & AWO NRW & West & $\mathrm{Kr} 9 \mathrm{~b}$ & 35.431 & 2.953 \\
\hline DRK-Reformtarif & DRK & bundesweit & $\mathrm{Kr} 9 \mathrm{~b}$ & 35.730 & 2.978 \\
\hline AVR Caritas & Caritas/Malteser & West & $\mathrm{Kr} 9 \mathrm{~b}$ & 36.662 & 3.055 \\
\hline AVR DW Bayern & Diakonie Bayern & West & EG 10 & 38.311 & 3.193 \\
\hline \multicolumn{6}{|l|}{$\begin{array}{l}\text { maximale } \\
\text { Erfahrungsstufe }\end{array}$} \\
\hline AVB & Paritätischer & West & GrE & 36.559 & 3.047 \\
\hline DRK-Reformtarif & DRK & bundesweit & $\mathrm{Kr} 9 \mathrm{~b}$ & 41.902 & 3.492 \\
\hline AVR DW Bayern & Diakonie Bayern & West & EG 10 & 42.470 & 3.539 \\
\hline TV AWO NRW & AWO NRW & West & $\mathrm{Kr} 9 \mathrm{~b}$ & 42.887 & 3.574 \\
\hline AVR Caritas & Caritas/Malteser & West & $\mathrm{Kr} 9 \mathrm{~b}$ & 44.205 & 3.684 \\
\hline
\end{tabular}

Quelle: Wohlfahrtintern-Tarifarchiv. 
TABELLE 6

Tarifliche Jahresgrundvergütung inkl. Urlaubsgeld und Jahressonderzahlung - Beschäftigte mit abgeschlossener Berufsausbildung Angaben in Euro

\begin{tabular}{|c|c|c|c|c|}
\hline Tätigkeit & Qualifikation/Anforderung & Vergütungsgruppe & 2010 & 2010 Monat \\
\hline \multicolumn{5}{|l|}{ Einstiegsvergütung } \\
\hline Altenpfleger/in & mit staatl. Anerkennung/Abschlussprüfung & TVöD EG 7a, Stufe 1 & 26.289 & 2.191 \\
\hline Krankenschwester/pfleger & mit staatl. Anerkennung & TVöD Kr. 7a, Stufe 1 & 26.612 & 2.218 \\
\hline Bankkaufmann/frau & Berufsausb. & 4 (Anf.-stufe) & 28.015 & 2.335 \\
\hline Erzieher/in & mit staatl. Anerkennung & TVöD S 6 Stufe 2 & 29.243 & 2.437 \\
\hline Versicherungskaufmann/frau & Berufsausb. & 3 (Anf.stufe) & 29.819 & 2.485 \\
\hline Metallbeschäftigte & Berufsausb. & E 7 & 38.544 & 3.212 \\
\hline \multicolumn{5}{|l|}{ Obere Vergütungen } \\
\hline Altenpfleger/in mind. 10 Pflegepersonen unterstellt & mit staatl. Anerkennung & TVöD EG 9b, Endstufe & 39.677 & 3.306 \\
\hline Leitung Krankenpflege mit mind. 25 Pflegepersonen & mit staatl. Anerkennung & TVöD EG c, Endstufe & 42.468 & 3.539 \\
\hline Metallbeschäftigte & Berufsausb. & E 9 & 43.941 & 3.662 \\
\hline Leiter/in Kita ab 40 Plätze. & mit staatl. Anerkennung & TVöD S 10, Stufe 6 & 44.819 & 3.735 \\
\hline Leitung Krankenpflege mit mind. 200 Pflegepersonen & mit staatl. Anerkennung & TVöD Kr.11a, Endstufe & 50.912 & 4.243 \\
\hline Metallbeschäftigte & Berufsausb. Experten-/Spezialistentätigkeit & E 12 & 53.381 & 4.448 \\
\hline Leiter/in Kita mind. 180 Kinder & mit staatl. Anerkennung & TVöD S 17 & 53.563 & 4.464 \\
\hline Bankkaufmann/frau & Berufsausb. & 9 (Endstufe) & 54.522 & 4.544 \\
\hline Versicherungskaufmann/frau & Berufsausb. & 8 (Endstufe) & 55.980 & 4.665 \\
\hline
\end{tabular}

TABELLE 7

Tarifliche Jahresgrundvergütung inkl. Urlaubsgeld und Jahressonderzahlung - Beschäftigte mit Fachhochschulabschluss Angaben in Euro

\begin{tabular}{|c|c|c|c|c|}
\hline Tätigkeit & Qualifikation/Anforderung & Vergütungsgruppe & 2010 & 2010 Monat \\
\hline \multicolumn{5}{|l|}{ Einstiegsvergütung } \\
\hline Sozialarbeiter/in & FH-Abschluss & TVöD S 11, Stufe 2 & 33.679 & 2.807 \\
\hline Metallbeschäftigte & FH-Abschluss & E 12 & 53.381 & 4.448 \\
\hline \multicolumn{5}{|l|}{ oberste Vergütung } \\
\hline Leitung Krankenpflege mind. 200 Pflegepersonen unterstellt & & TVöD Kr.11a, Endstufe & 50.912 & 4.243 \\
\hline Leiter/in Kita mind. $180 \mathrm{Ki}$. & & TVöD S 17 & 53.563 & 4.464 \\
\hline Bankgewerbe & Berufsausb. & 9 (Endstufe) & 54.522 & 4.544 \\
\hline Versicherungsgewerbe & Berufsausb. & 8 (Endstufe) & 55.980 & 4.665 \\
\hline Sozialarbeiter/in & FH-Abschluss & TVöD S 18 & 58.615 & 4.885 \\
\hline Metallbeschäftigte & FH-Abschluss & E 17 & 71.888 & 5.991 \\
\hline
\end{tabular}

kommen von rund 2.800 bis $5.900 €$ (Tabelle 7). Im Einstiegsbereich bewegt sich das Einkommen zwischen $33.700 €$ und etwa $53.400 €$. Die Differenz beträgt damit $58 \%$. Die Sozialarbeiter und Sozialarbeiterinnen erhalten die geringste Vergütung, die Beschäftigten der Metallindustrie die höchste. - Im oberen Einkommensbereich reichen die tariflichen Jahresvergütungen von knapp $51.000 €$ bis zu knapp $72.000 €$.
Hier startet die Reihe mit den Pflegeberufen (Leitung Krankenpflege mit mindestens 200 unterstellten Pflegepersonen), gefolgt von den Bank- und Versicherungskaufleuten und dahinter die Sozialarbeiterinnen und Sozialarbeiter. Mit deutlichem Abstand liegen die Beschäftigten der Metallindustrie an der Spitze. 
TABELLE 8

Tarifliche Jahresvergütung mit abgeschlossener Berufsausbildung - Einstiegsvergütung -

\begin{tabular}{lc|c|c|c}
\hline \multirow{2}{*}{ Tätigkeit } & \multirow{2}{*}{$\mathbf{1 9 9 5}$} & 2010 & \multicolumn{2}{c}{ Steigerung } \\
& $€$ & $€$ & in $€$ & in \% \\
\hline Metallfacharbeiter/in & 23.006 & 38.544 & 15.538 & 67,5 \\
\hline Versicherungskaufmann/-frau & 22.366 & 29.819 & 7.453 & 33,3 \\
\hline Erzieher/in & 21.231 & 29.243 & 8.011 & 37,7 \\
\hline Bankkaufmann/frau & 20.924 & 28.015 & 7.091 & 33,9 \\
\hline Krankenschwester & 21.912 & 26.612 & 4.700 & 21,4 \\
\hline Altenpfleger/in & 21.912 & 26.289 & 4.377 & 20,0 \\
\hline
\end{tabular}

Quelle:WSI-Tarifarchiv.

\section{Fazit}

Der Anstieg der Tarifvergütungen von 1995 - 2010 variiert sehr stark zwischen den Branchen: An der Spitze liegt die Metallindustrie mit einem Plus von durchschnittlich $55 \%$, gefolgt vom Bankgewerbe und Versicherungsgewerbe mit jeweils einem Anstieg von 33-34 \%. Im öffentlichen Dienst ist dagegen nur ein durchschnittlicher Anstieg um gut $22 \%$ zu verzeichnen. Dadurch erhöhen sich die Tariflohndifferenzen zwischen den untersuchten Branchen weiter.

Tabelle 8 verdeutlicht die Entwicklung am Beispiel der Tätigkeiten mit abgeschlossener Berufsausbildung in der Einstiegsstufe. Die gezeigten Relationen und Rangfolgen lassen sich allerdings keineswegs auf alle Tätigkeiten und Erfahrungsstufen übertragen. In der Metallindustrie und im öffentlichen Dienst ergeben sich allerdings sehr starke Unterschiede zwischen den einzelnen Beschäftigtengruppen infolge der Entgeltstrukturreformen im Rahmen von ERA und TVöD, die die vormals völlig getrennten Lohn- und Gehaltstarifverträge modernisiert und zu einheitlichen Entgelttarifverträgen zusammengeführt haben.

Zusammenfassend ist festzuhalten: Die tariflichen Jahreseinkommen von Beschäftigten mit abgeschlossener Berufsausbildung bzw. mit Fach- hochschulqualifikation in der Metallindustrie, im Bankgewerbe, im Versicherungsgewerbe, im Bereich Gesundheitswesen/Pflege sowie im Sozialund Erziehungsdienst weisen erhebliche Unterschiede auf, die sich im Laufe des Beobachtungszeitraums von 15 Jahren in der Tendenz vergrößert haben. Familienbezogene Vergütungselemente, die zu Beginn des Beobachtungszeitraums vor allem im öffentlichen Dienst und im Finanzdienstleistungsbereich bestanden, existieren heute nicht mehr bzw. allenfalls noch als auslaufende Besitzstandsregelungen. Die tariflichen Einkommensniveaus im Bereich der Metallindustrie sowie bei den Finanzdienstleistern liegen in der Regel deutlich höher als im Gesundheits- und Sozialbereich. Eine Ausnahme stellen obere Leitungspositionen im Gesundheits- und Sozialbereich dar, wo im Einzelfall auch das Vergütungsniveau der Industrie bzw. von Banken und Versicherungen erreicht wird. Allerdings handelt es sich dabei im Vergleich zur Gesamtzahl der Arbeitsplätze in diesem Bereich vermutlich nur um eine sehr geringe Anzahl.

Insgesamt zeigt sich in der vergleichenden Betrachtung der Tariflohnentwicklung in Westdeutschland nach Branchen, dass gegenwärtig in der Metallindustrie als einem industriellen Kernsektor, in dem zugleich überwiegend Männer beschäftigt sind, wie auch in dem gemischt geschlechtlich besetzten Banken- und Versicherungsbereich deutlich höhere Tariflöhne erzielt werden als in den durch öffentliche oder quasi-öffentliche Beschäftigung gekennzeichneten und frauendominierten Gesundheits- und Sozialbereichen. Diese Differenz hat sich im Zeitverlauf tendenziell verstärkt.

\section{AUTOREN}

REINHARD BISPINCK, Dr., ist Wissenschaftler im Wirtschafts- und Sozialwissenschaftlichen Institut (WSI) und Leiter des WSI-Tarifarchivs in der HansBöckler-Stiftung.

reinhard-bispinck@boeckler.de

GÖTZ BAUER, ULRICH SCHMIDT, MONIKA SCHWACKE-PILGER, KATHRIN SONNEN, ANDREA TAUBE und MONIKA WIEBEL sind Sachbearbeiterinnen und Sachbearbeiter im WSI-Tarifarchiv. 